\title{
Effectiveness of surgical ventricular restoration in patients with dilated ischemic cardiomyopathy and unrepaired mild mitral regurgitation
}

Marisa Di Donato, MD, ${ }^{a}$ Serenella Castelvecchio, MD, Jelena Brankovic, MD, ${ }^{\mathrm{b}}$ Carlo Santambrogio, MD, Vincenzo Montericcio, MD, ${ }^{\mathrm{b}}$ and Lorenzo Menicanti, $\mathrm{MD}^{\mathrm{b}}$

From the Department of Critical Care Medicine, University of Florence, ${ }^{\mathrm{a}}$ Florence, Italy; and Department of Cardiac Surgery, San Donato Hospital, ${ }^{\text {b }}$ Milan, Italy.

Received for publication May 17, 2007; revisions received July 30, 2007; accepted for publication Aug 16, 2007.

Address for reprints: Marisa Di Donato, MD, Department of Cardiac Surgery, San Donato Hospital, Via Morandi 30, 20097 San Donato Milanese (Milan), Italy (E-mail: marad@ tin.it).

J Thorac Cardiovasc Surg 2007;134:1548-53 $0022-5223 / \$ 32.00$

Copyright $\odot 2007$ by The American Association for Thoracic Surgery

doi:10.1016/j.jtcvs.2007.08.031
Objective: Any grade of ischemic mitral regurgitation is associated with excess mortality. Whether mild ischemic mitral regurgitation should be repaired at the time of either coronary artery bypass grafting or surgical ventricular restoration is controversial. Surgical ventricular restoration is a treatment option for dilated post-infarction cardiomyopathy and has the potential to improve mitral functioning. The present study assessed the effectiveness of surgical ventricular restoration and unrepaired mild ischemic mitral regurgitation on left ventricular geometry, cardiac and functional status, and survival.

Methods: We analyzed 55 patients with previous anterior infarction (age $65 \pm 10$ years) and mild chronic functional mitral regurgitation who underwent surgical ventricular restoration and coronary artery bypass grafting without mitral repair at our center. Left ventricular volumes, ejection fraction, and geometric parameters were measured before and after surgery.

Results: Even mild ischemic mitral regurgitation is characterized by abnormal left ventricular geometry when compared with that of patients without mitral regurgitation at comparable ventricular volumes and ejection fraction. Surgical ventricular restoration induces a significant decrease in left ventricular volumes, left ventricular diameters, and papillary muscle distance; and an improvement in ejection fraction and New York Heart Association class. Ischemic mitral regurgitation significantly decreases in the majority of patients. Survival is $93 \%$ at 1 year and $88 \%$ at 3 years.

Conclusion: Surgical ventricular restoration improves mitral functioning by improving geometry abnormalities. Survival is optimal and greater than would be expected in patients with post-infarction dilated ventricles and depressed left ventricular function. Our data indicate that mitral repair in conjunction with surgical ventricular restoration is unnecessary in such patients.

I schemic mitral regurgitation (IMR) is frequently associated with dilated postinfarction cardiomyopathy and indicates a poor survival, even when mild. ${ }^{1,2}$ Surgical ventricular restoration (SVR) aims to restore normal left ventricular (LV) geometry and function and includes complete coronary revascularization, ventricular reconstruction, and mitral repair when needed. ${ }^{3-7}$ Although there is general agreement that IMR should be treated surgically when it is moderate to severe, there is no consensus if, how, and when mild IMR should be repaired in conjunction with SVR. SVR has 3 important objectives to alter functional mitral regurgitation (MR); these are directed toward the vessel, ventricle, and valve, and therefore has the potential of improving mitral valve functioning by reducing LV volume, reapproaching papillary muscles, and rebuilding a more normal geometry. ${ }^{8-11}$ Some authors recommend treating the mitral valve in conjunction with SVR, ${ }^{9,12}$ but data on the natural history and surgical results are limited. 


$$
\begin{aligned}
& \text { Abbreviations and Acronyms } \\
& \qquad \begin{aligned}
\mathrm{CABG} & =\text { coronary artery bypass grafting } \\
\mathrm{EF} & =\text { ejection fraction } \\
\mathrm{ERO} & =\text { effective regurgitant orifice } \\
\mathrm{IMR} & =\text { ischemic mitral regurgitation } \\
\mathrm{LV} & =\text { left ventricular } \\
\mathrm{MR} & =\text { mitral regurgitation } \\
\mathrm{SVR} & =\text { surgical ventricular restoration }
\end{aligned}
\end{aligned}
$$

The objective of the present study was to assess the effectiveness of SVR in patients with unrepaired mild IMR who underwent surgery for dilated ischemic cardiomyopathy caused by anterior infarction. To define geometric characteristics in terms of sphericity, annular size, and papillary muscle width, we used comparisons in patients with anterior infarction but without MR.

\section{Materials and Methods Patient Selection}

From July of 2001 to December of 2006, 309 consecutive patients with previous anterior myocardial infarction and symptoms of heart failure, angina, or a combination of the two underwent SVR at our center. Clinical, demographic, and echocardiographic data were prospectively gathered and stored on a customized database that had been approved by our local ethical committee. In our patients, we elect to repair the mitral valve in conjunction with SVR when IMR is moderate to severe (grade 3-4+) and in mild IMR (grade 2+) when the mitral annulus is dilated ( $\geq 40 \mathrm{~mm}$ ). Among 64 patients with grade 2 IMR, 55 did not undergo repair and they represent our study group.

All patients had complete transthoracic echocardiograms before surgery and at discharge (8-10 days after surgery). To define geometric characteristics in sphericity, annular size, and papillary muscle width, we used comparisons in 67 patients (aged $65 \pm 9$ years, 11 female) with anterior infarction but without MR. Cardiac operative mortality was defined as death from cardiac causes occurring within 30 days from surgery; long-term mortality includes death from any cause.

\section{Echocardiographic Studies}

Two-dimensional, M-mode, and color Doppler transthoracic echocardiography was performed using a GE Vivid 7 (GE Healthcare, Waukesha, Wis) echocardiographic instrument, and all 4-chamber, 2-chamber, long-axis, and short-axis views were analyzed before and after surgery in all patients. The parameters measured included the following:

- Diastolic and systolic internal diameters in parasternal longaxis view (millimeters)

- End-diastolic and end-systolic volume calculated by applying the Simpson method (milliliters)

- Ejection fraction (EF) derived from volumes as end-diastolic volume - end-systolic volume/end-diastolic volume (\%)

- Mitral annulus dimension defined as the distance between the hinge points of the anterior and posterior mitral leaflets in long-axis view (millimeters)
- Papillary muscle distance in short-axis view defined as the distance between the 2 papillary muscle heads (millimeters)

- Long axis measured in 4-chamber view as the distance between the apex and the mitral plane (millimeters)

- Short axis measured in 4-chamber view at the midlevel of the long axis (millimeter)

- Sphericity index calculated as the short- to long-axis ratio, in diastole and systole

In our daily clinical activity, we assess the degree of MR in a semiquantitative way using the following scale: $0=$ absent, $1+=$ trivial, $2+=$ mild, $3+=$ moderate, and $4+=$ severe.

\section{Patient Characteristics}

There were 9 women and 46 men with a mean age of $65 \pm 10$ years. All had grade 2+ MR; 29 patients had New York Heart Association class 3 or $4 ; 30$ patients had a preoperative EF less than $35 \%$.

\section{Surgical Technique}

The details of the surgical technique have been reported. $4,5,7,8,10$ Briefly, the procedure is conducted on the arrested heart with antegrade crystalloid or blood cold cardioplegia. Complete coronary artery revascularization is first performed, almost always with the left internal thoracic artery on the left anterior descending artery and sequential venous grafts on the right and circumflex arteries, when needed. After completion of coronary grafting, the left ventricle is opened with an incision parallel to the left anterior descending artery, extending from the apex to the middle scarred region. The ventricular cavity is inspected, and thrombi are removed if they are present. Each papillary muscle head is identified, and the mitral valve leaflets and chords are evaluated. SVR is performed using a mannequin (TRISVR, Chase Medical, Richardson, Tex) filled at 50 to $60 \mathrm{~mL} / \mathrm{m}^{2}$ to optimize the size and shape of the new ventricle.

\section{Results}

All patients underwent SVR, and all patients except 2 had concomitant coronary artery bypass grafting (CABG). The mean number of distal anastomosis was $2.9 \pm 1.4$; preoperative patient characteristics and medications are summarized in Table 1. The operative cardiac mortality was $7.2 \%$ (4/55). Univariate analysis showed that patients who died had significantly lower EF $(27 \% \pm 8 \%$ vs $35 \% \pm 7 \%$; $P=.009)$, thinner interventricular septum $(9 \pm 2.0 \mathrm{~mm}$ vs $11 \pm 2.8 \mathrm{~mm} ; P=.01)$, greater QRS (138 $\pm 26 \mathrm{msec}$ vs $112 \pm 27 \mathrm{msec} ; P=.01)$, and greater pulmonary pressure $(47 \pm 11 \mathrm{~mm} \mathrm{Hg}$ vs $35 \pm 9 \mathrm{~mm} \mathrm{Hg} ; P=.02)$. The cause of operative mortality was low cardiac output in all patients. Table 2 shows the operative data and early postoperative complications.

Table 3 shows the baseline geometric measurements in patients with MR compared with patients without MR. Volumes and EF are not significantly different; geometric measurements are significantly abnormal with respect to patients without MR. This finding indicates that geometric 
TABLE 1. Preoperative patient characteristics and medications $(n=55)$

\begin{tabular}{lcl}
\hline & $\mathbf{n}$ & $(\%)$ \\
\hline Male/female & $46 / 9$ & \\
Age & $65 \pm 10$ & \\
Diabetes & 17 & $(31)$ \\
Impaired renal function & 7 & $(13)$ \\
Atrial fibrillation & 7 & $(13)$ \\
Cerebrovascular events & 4 & $(7)$ \\
Previous PTCA & 6 & $(11)$ \\
Previous CABG & 2 & $(4)$ \\
Previous ICD & 4 & $(7)$ \\
Angina & 40 & $(75)$ \\
Unstable & 10 & $(18)$ \\
NYHA class III/IV & 29 & $(53)$ \\
Current medications & & \\
$\quad$ ACEls & 45 & $(82)$ \\
Beta-blockers & 34 & $(62)$ \\
Aspirin & 47 & $(84)$ \\
Warfarin & 3 & $(5)$ \\
Diuretics & 36 & $(65)$ \\
Nitrates & 29 & $(53)$ \\
Statins & 23 & $(42)$ \\
Digoxin & 2 & $(4)$ \\
\hline
\end{tabular}

PTCA, Percutaneous transluminal coronary angiography; $C A B G$, coronary artery bypass grafting; $I C D$, implantable cardioverter defibrillator; $N Y H A$, New York Heart Association; ACEl, angiotensin-converting enzyme inhibitor.

abnormalities, and not volume increase, are major determinants of MR in patients with anterior infarction.

\section{Early Functional and Geometric Changes Induced by Surgical Ventricular Restoration}

Transthoracic echocardiography performed at discharge (8-10 days after surgery) was used for all postoperative measurements. The results are shown in Table 4. There is a significant reduction in dimensions and volumes, both in diastole and systole, an improvement in $\mathrm{EF}$, a reduction in papillary muscle distance, and no change in mitral annulus or sphericity index.

The average degree of MR after surgery was $1 \pm 0.8$; it was unchanged in 12 patients, improved in 36 patients, and increased to grade $3+$ in 3 patients (Figure 1). Patients without improved MR after SVR had significantly greater diastolic and systolic diameters preoperatively and postoperative; both volumes and diameters were greater compared with those of patients with improved mitral functioning (Table 5). We could not determine a cutoff value of diastolic or systolic LV dimensions predictive of lack of improvement in mitral functioning or adverse events; however, if the diastolic internal diameter or the short axis exceeds $65 \mathrm{~mm}$, it is unlikely that MR can improve without concomitant mitral surgery.
TABLE 2. Operative data and early postoperative complications

\begin{tabular}{lcc}
\hline & $\mathbf{n}$ & $\%$ \\
\hline Multivessel CAD & 41 & 74 \\
CABG & 53 & 96 \\
ITA/LAD & 51 & 93 \\
Distal anastomosis & $2.9 \pm 1.4$ & \\
Complications & & \\
$\quad$ Bleeding & 1 & 2.6 \\
Infections & 7 & 13 \\
Renal failure & 2 & 3.6 \\
IABP & 5 & 9 \\
TIA & 2 & 3.6 \\
VA & 4 & 7 \\
AF & 7 & 12.7
\end{tabular}

$C A D$, Coronary artery disease; $C A B G$, coronary artery bypass grafting; ITA, internal thoracic artery; $L A D$, left anterior descending artery; $I A B P$, intraaortic balloon pumping; TIA, transitory ischemic attack; $V A$, ventricular arrhythmias; $A F$, atrial fibrillation.

\section{Late Findings}

New York Heart Association class improved from $2.5 \pm 0.7$ to $1.4 \pm 0.5(P=.0001)$. Twenty patients had a new echo evaluation late after surgery (mean time from surgery was 12 months, minimum of 4 months and maximum of 37 months). Seven patients had no or trivial MR, 10 patients had grade $2 \mathrm{MR}$, and 3 patients had grade $3+\mathrm{MR}$.

Follow-up was $100 \%$ complete. Mean follow-up was $25 \pm 13$ months. There were 10 hospital readmissions during the entire follow-up: 3 for heart failure, 1 for a transitory ischemic attack, 3 for noncardiac causes, and 1 for arrhythmia. One patient received an implantable cardioverter defibrillator, and 1 patient received an implantable cardioverter defibrillator plus cardiac resynchronization therapy. One patient died of a noncardiac cause at 28 months. The actuarial survival curve, including operative mortality, is shown in Figure 2. Overall survival, including early mortality, was $93 \%$ at 1 year and $88 \%$ at 3 years.

\section{Discussion}

The objective of this study was to assess the effectiveness of SVR in patients undergoing SVR and unrepaired mild ischemic MR. To ensure a homogeneous series of patients, we specifically studied the impact of mild MR after anterior myocardial infarction because the underlying ischemic disease does not directly affect the papillary muscles, leaflets, chords, or annulus, and MR is consequently related completely to LV remodeling. In previous articles, ${ }^{4.10}$ we reported that any degree of MR in anterior infarction resulted in 1) worsening of clinical and hemodynamic parameters, 2) shape and function abnormalities in remote inferior wall, and 3) worsening in volume and geometry in untreated MR. We also speculated that it might be unwise to leave any 
TABLE 3. Baseline geometric measurements in mitral regurgitation grade $2+$ compared with those in no mitral regurgitation

\begin{tabular}{lccc}
\hline & No MR $(\mathbf{n}=\mathbf{6 7})$ & MR grade 2+ (n= 55) & P \\
\hline Diastolic diameter (mm) & $58 \pm 9$ & $64 \pm 8$ & .009 \\
Systolic diameter (mm) & $46 \pm 10$ & $52 \pm 11$ & .01 \\
End-diastolic volume (mL) & $191 \pm 51$ & $201 \pm 69$ & $\mathrm{NS}$ \\
End-systolic volume (mL) & $127 \pm 44$ & $137 \pm 60$ & $\mathrm{NS}$ \\
EF (\%) & $34 \pm 9$ & $34 \pm 8$ & $\mathrm{NS}$ \\
Mitral annulus (mm) & $30 \pm 4$ & $34 \pm 4$ & .02 \\
Diastolic short axis (mm) & $49 \pm 8$ & $54 \pm 8$ & .05 \\
Systolic short axis (mm) & $38 \pm 9$ & $47 \pm 8$ & .003 \\
Papillary muscle dist (mm) Diastole & $24 \pm 8$ & $28 \pm 5$ & .02 \\
Sphericity index (diastole) & $0.48 \pm 0.08$ & $0.56 \pm 0.09$ & .003 \\
Sphericity index (systole) & $0.40 \pm 0.09$ & $0.47 \pm 0.01$
\end{tabular}

$M R$, Mitral regurgitation; $E F$, ejection fraction; $N S$, not significant.

functional MR in large ventricles with a wide transverse diameter and hoped that future studies would test the concept. Data on the natural history of functional MR are limited, but several observational studies showed that MR has an independent association with a poor prognosis.

\section{Ischemic Mitral Regurgitation}

A clear definition of IMR is lacking, and different descriptors have resulted in heterogeneous patient groups, which in turn complicate comparisons between studies. ${ }^{3}$

In ischemic MR, the mitral leaflets are normal and MR is caused by deformation of subvalvular mitral apparatus as the result of LV remodeling. Echocardiographic characteristics of ischemic MR include low mitral leaflet coaptation and anterior and posterior leaflet tethering causing restriction of mitral leaflets with or without concomitant wall motion abnormalities, particularly at the posterolateral wall. ${ }^{13} \mathrm{LV}$ distortion and remodeling after an anterior infarction displace papillary muscle toward the apex, resulting in apical mitral tethering and restriction of coaptation during

TABLE 4. Preoperative and postoperative data in patients with mitral regurgitation

\begin{tabular}{lccl}
\hline & Preoperative & Postoperative & $\boldsymbol{P}$ \\
\hline Diastolic diameter (mm) & $64 \pm 8$ & $61 \pm 9$ & .007 \\
Systolic diameter (mm) & $52 \pm 11$ & $48 \pm 9$ & .01 \\
End-diastolic volume (mL) & $201 \pm 69$ & $150 \pm 49$ & .0001 \\
End-systolic volume (mL) & $137 \pm 60$ & $94 \pm 39$ & .0001 \\
EF (\%) & $34 \pm 8$ & $41 \pm 8$ & .0001 \\
Mitral annulus (mm) & $34 \pm 4$ & $33 \pm 7$ & NS \\
Diastolic short axis (mm) & $54 \pm 8$ & $49 \pm 6$ & .0005 \\
Systolic short axis (mm) & $47 \pm 8$ & $42 \pm 8$ & .001 \\
Papillary muscle dist (mm) (D) & $28 \pm 5$ & $25 \pm 7$ & .02 \\
Sphericity index (diastole) & $0.56 \pm 0.09$ & $0.58 \pm 0.08$ & $\mathrm{NS}$ \\
Sphericity index (systole) & $0.47 \pm 0.01$ & $0.51 \pm 0.14$ & $\mathrm{NS}$ \\
\hline
\end{tabular}

$E F$, Ejection fraction; NS, not significant. systole; the reduced contractile function decreases the closing forces on the leaflets. ${ }^{14-19}$ The MR jet is usually central, and the severity is often mild. In these patients, conservative management is associated with poor prognosis with a 1-year survival of $30 \%$ to $40 \% .^{20}$ The excess mortality is independent of baseline characteristics and degree of ventricular dysfunction; the effective regurgitant orifice (ERO) was the only independent predictor of mortality. ${ }^{1}$ An ERO less than or equal to $20 \mathrm{~mm}^{2}$ identifies mild MR, but despite its negative impact on survival there is no consensus on whether and when to intervene in mild MR in patients who are undergoing $\mathrm{CABG}$ alone or associated with SVR. A study in patients undergoing isolated $\mathrm{CABG}$ without severe IMR reported that the presence of moderate IMR, and even mild IMR, was associated with decreased survival. ${ }^{21}$ SVR has the potential of improving mitral functioning, but there are limited data on the effectiveness of SVR on unrepaired IMR.

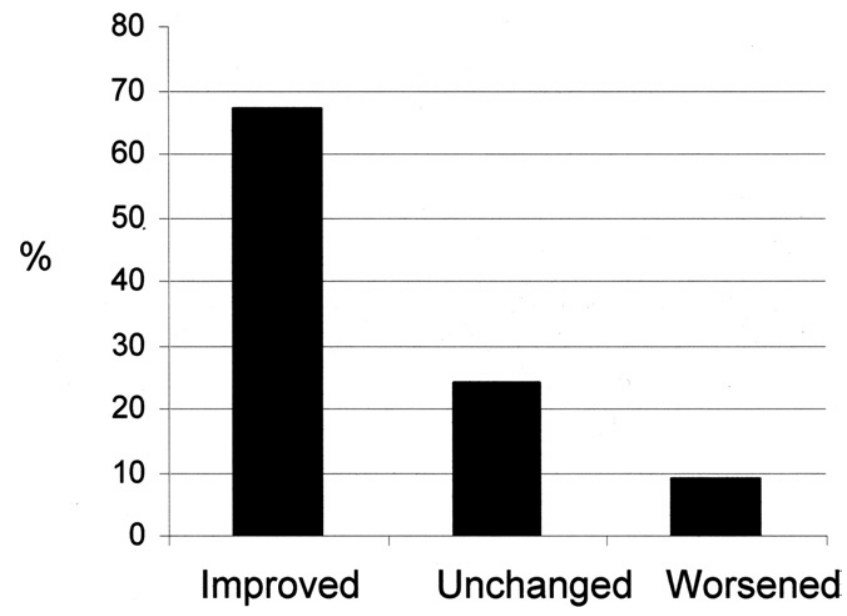

Figure 1. Changes induced by SVR in mitral functioning. SVR, Surgical ventricular restoration. 
TABLE 5. Patient characteristics by surgical ventricular restoration-induced changes in mitral functioning

\begin{tabular}{|c|c|c|c|c|c|c|c|c|c|c|}
\hline & \multicolumn{5}{|c|}{ Preoperative Data } & \multicolumn{5}{|c|}{ Postoperative Data } \\
\hline & DD & SD & EDV & ESV & EF & DD & SD & EDV & ESV & EF \\
\hline MR improved $\mathrm{N}=36$ & $61 \pm 9$ & $46 \pm 10$ & $199 \pm 84$ & $137 \pm 78$ & $34 \pm 8$ & $54 \pm 7$ & $43 \pm 8$ & $120 \pm 31$ & $70 \pm 23$ & $42 \pm 8$ \\
\hline $\begin{array}{l}\text { MR not improved } \mathrm{N}=15 \\
P\end{array}$ & $\begin{array}{c}67 \pm 6 \\
.03\end{array}$ & $\begin{array}{c}53 \pm 7 \\
.03\end{array}$ & $\begin{array}{l}209 \pm 55 \\
\quad N S\end{array}$ & $\begin{array}{l}142 \pm 45 \\
\mathrm{NS}\end{array}$ & $\begin{array}{c}33 \pm 6 \\
\mathrm{NS}\end{array}$ & $\begin{array}{l}61 \pm 6 \\
.003\end{array}$ & $\begin{array}{c}50 \pm 7 \\
.01\end{array}$ & $\begin{array}{c}140 \pm 43 \\
.05\end{array}$ & $\begin{array}{c}86 \pm 27 \\
.04\end{array}$ & $\begin{array}{c}39 \pm 5 \\
\text { NS }\end{array}$ \\
\hline
\end{tabular}

$\overline{D D}$, Diastolic diameter $(\mathrm{mm}) ; S D$, systolic diameter $(\mathrm{mm}) ; E D V$, end-diastolic volume $(\mathrm{mL}) ; E S V$, end-systolic volume (mL); $E F$, ejection fraction $(\%) ; N S$, not significant; $M R$, mitral regurgitation.

It is generally accepted that moderate to severe MR (grade 3-4+) is an indication for surgical repair in conjunction with SVR, and it has been reported that adding mitral repair to SVR $\pm \mathrm{CABG}$ and to $\mathrm{CABG}$ alone increases the operative risk because of the prolonged surgical time, technical complexity, and patient selection..$^{3,22-24}$ In our experience we elect to repair the mitral valve through a ventriculotomy with a posterior suture during SVR, when the degree of MR is moderate or severe (grade 3-4+), and, if the degree is mild, we perform mitral repair if the annulus is markedly dilated $(\geq 40 \mathrm{~mm}$ ). In our series we found 9 patients with grade 2 MR and annular dilatation greater than $40 \mathrm{~mm}$ who had mitral repair. Such a small number precludes any comment on this peculiar subset of patients. A recent article by Sartipy and colleagues ${ }^{23}$ confirmed a higher operative mortality (16\%) in patients with mild-tosevere MR undergoing mitral repair in conjunction with SVR, and survival in these patients was significantly worse than in patients undergoing SVR without mitral repair. In the series by Sartipy and coworkers, the degree of repaired MR was mild (2+) in the majority of patients (18/31), and they used the same semiquantitative assessment of MR as we did. Our results, showing an optimal midterm survival

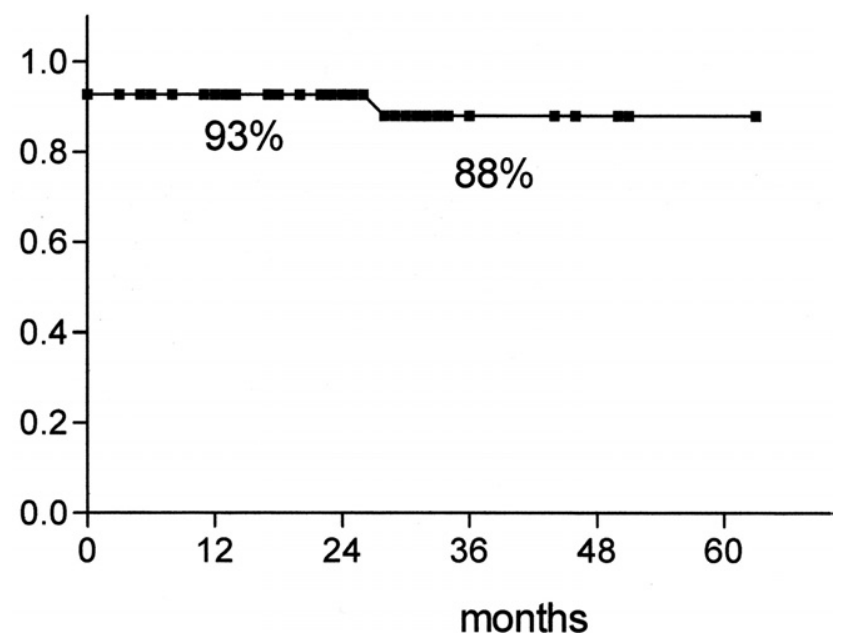

Figure 2. Survival curve after SVR in patients with unrepaired mild ischemic MR. SVR, Surgical ventricular restoration; MR, mitral regurgitation. and an acceptable operative risk in patients with dilated ventricles, reduced LV function, and unrepaired mild MR, suggest that mitral repair in conjunction with SVR is unnecessary in such patients. Survival in our patient population is higher than that expected in the natural history of patients postinfarction with chronic IMR. ${ }^{1,2}$

\section{Left Ventricular Geometry in Patients With Ischemic Mitral Regurgitation and Effectiveness of Surgical Ventricular Restoration}

MR in organic mitral disease is considered severe when the ERO is greater than $40 \mathrm{~mm}^{2}$, but in functional ischemic MR a lower threshold of regurgitant orifice $(E R O \geq 20)$ is sufficient to diagnose severe MR because of its association with poor prognosis. ${ }^{1,3}$ In our patients with mild MR, LV volumes are large, EF is depressed, and geometry is abnormal. SVR improves geometry and function and reduces the degree of MR in the majority of patients. Patients without improved mitral functioning have larger transverse diameters preoperatively, and despite SVR reduction, MR does not improve. A larger population is needed to find predictors of lack of mitral improvement. However, the residual mild MR (only 3 patients with grade $3+$ ) does not seem to affect New York Heart Association class improvement or survival.

Our data confirm that IMR is a ventricle and not a valve pathologic condition. The reconstruction of the ventricle leads to a better functioning of the mitral valve by reducing papillary muscle distance (which is a main determinant of functional MR) and LV volumes. Sphericity does not change significantly, and this is because of the greater reduction of the long axis with respect to the short axis, despite the use of the shaper device that helps to maintain a more normal short- to long-axis ratio. The improvement in cardiac and functional status and in mitral functioning led to an optimal survival in our series of patients.

\section{Limitations}

The major limitation is that this study is observational and not randomized. We report the results from our clinical program and did not include an appropriate control group of patients who underwent the mitral procedure, because we have only 9 such patients and cannot draw any conclusions at present. Survival in our population is good if we consider 
that worsening heart failure symptoms and a poor prognosis would be expected for such patients. ${ }^{24-27}$ Another limitation is that the severity of MR was estimated in a semiquantitative way by color Doppler 2-dimensional echo technique, which is the most common MR evaluation obtained in a cardiac surgery setting. Finally, mitral assessment late after surgery is limited and not protocol driven.

\section{Conclusions}

Our study shows that SVR associated with complete coronary revascularization in the setting of modest LV dysfunction, dilated ventricles, and mild MR improves mitral functioning, at least initially, by correcting the abnormal LV geometry and relieving ischemia. The rate of hospital readmission is low; only 2 patients required implantable cardioverter defibrillator and cardiac resynchronization therapy post SVR. Midterm survival is optimal and considerably higher than that reported in patients after myocardial infarction with any grade of IMR.

\section{References}

1. Grigioni F, Enriquez-Sarano M, Zehr KJ, Bailey KR, Tajik AJ. Ischemic mitral regurgitation: long-term outcome and prognostic implications with quantitative Doppler assessment. Circulation. 2001;103: 1759-64.

2. Lamas GA, Mitchell GF, Flaker GC, Smith SC Jr, Gersh BJ, Basta L, et al. Clinical significance of mitral regurgitation after acute myocardial infarction: survival and Ventricular Enlargement Investigators. Circulation. 1997;96:827-33.

3. Borger MA, Alam A, Murphy PM, Doenst T, David TE. Chronic ischemic mitral regurgitation: repair, replace or rethink? Ann Thorac Surg. 2006;81:1153-61.

4. Athanasuleas CL, Buckberg GD, Stanley AWH, Siler W, Dor V, Di Donato M, et al., Restore group. Surgical ventricular restoration in the treatment of congestive heart failure due to post-infarction ventricular dilation. J Am Coll Cardiol. 2004;44:1439-45.

5. Menicanti L, Di Donato M. The Dor procedure: what has changed after fifteen years of clinical practice? J Thorac Cardiovasc Surg. 2002; 124:886-90.

6. Di Donato M, Frigiola A, Benhamouda M, Menicanti L. Safety and efficacy of surgical ventricular restoration in unstable patients with recent anterior myocardial infarction. Circulation. 2004;110(11 Suppl 1): II169-73.

7. Dor V, Sabatier M, Montiglio F, Coste P, Di Donato M. Endoventricular patch reconstruction in large ischemic wall-motion abnormalities. J Card Surg. 1999;14:46-52.

8. Menicanti L, Di Donato M, Frigiola A, Buckberg G, Santambrogio C, Ranucci M, et al. Ischemic mitral regurgitation: intraventricular papillary muscle imbrication without mitral ring during left ventricular restoration. J Thorac Cardiovasc Surg. 2002;123:1041-50.

9. Qin JX, Shiota T, McCarthy PM, Asher CR, Hail M, Agler DA, et al. Importance of mitral valve repair associated with left ventricular reconstruction for patients with ischemic cardiomyopathy: a realtime three-dimensional echocardiographic study. Circulation. 2003; 108(suppl II):II-241-6.
10. Menicanti L, Di Donato M, Castelvecchio S, Santambrogio C, Montericco V, Frigiola et al. Functional ischemic mitral regurgitation in anterior ventricular remodeling: results of surgical ventricular restoration with and without mitral repair. Heart Fail Rev. 2004;9:317-27.

11. Mickleborough LL, Merchant N, Ivanov J, Rao V, Carson S. Left ventricular reconstruction: early and late results. J Thorac Cardiovasc Surg. 2004;128:27-37.

12. Tulner SA, Steendijk P, Klautz RJ, Tops L, Bax J, Versteegh MI, et al. Clinical efficacy of surgical heart failure therapy by ventricular restoration and restrictive mitral annuloplasty. J Card Fail. 2007;13: $178-83$.

13. Izumi S, Miyatake K, Beppu S, Park YD, Nagata S, Kinoshita N, et al. Mechanism of mitral regurgitation in patients with myocardial infarction, a study using a real time two dimensional Doppler flow imaging and echocardiography. Circulation. 1987;76:777-85.

14. Yiu SF, Enriquez-Sarano M, Tribouilloy C, Seward JB, Tajik AJ. Determinants of the degree of functional mitral regurgitation in patients with systolic left ventricular dysfunction: a quantitative clinical study. Circulation. 2000;102:1400-6.

15. Kaul S, Spotnitz WD, Glasheen WP, Touchstone DA. Mechanism of ischemic mitral regurgitation; an experimental evaluation. Circulation. 1991;84:2167-80.

16. Tibayan FA, Rodriguez F, Zasio MK, Bailey L, Liang D, Daughters GT, et al. Geometric distortions of the mitral valvular-ventricular complex in chronic ischemic mitral regurgitation. Circulation. 2003; 108(Suppl II):II116-21.

17. Kwan J, Shiota T, Agler DA, Popovic ZB, Qin JX, Gillinov MA, et al. Geometric differences of the mitral apparatus between ischemic and dilated cardiomyopathy with significant mitral regurgitation: real-time three-dimensional echocardiography study. Circulation. 2003;107: 1135-40.

18. Levine RA, Hunk J. Ischemic mitral regurgitation, the dynamic lesion: clues to the cure. J Am Coll Cardiol. 2003;42:1929-32.

19. Levine RA. Dynamic mitral regurgitation: more than meets the eye. N Engl J Med. 2004;351:1681-4.

20. Naqvi TZ. Perioperative clinical decision making for mitral valve repair. Minerva Cardioangiol. 2007;55:213-27.

21. Grossi EA, Crooke GA, DiGiorgi PL, Schwartz CF, Jorde U, Applebaum RM, et al. Impact of moderate functional mitral insufficiency in patients undergoing surgical revascularization. Circulation. 2006; 114(Suppl I):I-573-6.

22. Duarte IG, Shen Y, MacDonald MJ, Jones EL, Craver JM, Guyton RA. Treatment of moderate mitral regurgitation and coronary disease by coronary bypass alone: late results. Ann Thorac Surg. 1999;68:426-30.

23. Sartipy U, Albåge A, Mattsson E, Lindblom D. Edge-to-edge mitral repair without annuloplasty in combination with surgical ventricular restoration. Ann Thorac Surg. 2007;83:1303-9.

24. Mallidi HR, Pelletier MP, Lamb J, Desai N, Sever J, Christakis GT, et al. Late outcomes in patients with uncorrected mild to moderate mitral regurgitation at the time of isolated coronary artery bypass grafting. J Thorac Cardiovasc Surg. 2004;127:636-44.

25. Stevenson LW, Fowler MB, Schroeder JS, Stevenson WG, Dracup KA, Fond V. Poor survival of patients with idiopathic cardiomyopathy considered too well for transplantation. Am J Med. 1987;83:871-6.

26. Koelling TM, Aaronson KD, Cody RJ, Bach DS, Armstrong WF. Prognostic significance of mitral regurgitation and tricuspid regurgitation in patients with left ventricular systolic dysfunction. Am Heart J. 2002;144:524-9.

27. Trichon BH, Felker GM, Shaw LK, Cabell CH, O'Connor CM. Relation of frequency and severity of mitral regurgitation to survival among patients with left ventricular systolic dysfunction and heart failure. Am J Cardiol. 2003;91:538-43. 